\title{
Emerging therapies for malignant pleural mesothelioma
}

\author{
Josine M. M. F. Quispel-Janssen • Paul Baas
}

Published online: 8 March 2012

(C) Springer Science+Business Media, LLC 2012

\begin{abstract}
Malignant pleural mesothelioma continues to challenge clinicians and scientists, since its incidence is rising and prognosis is far from favorable. Currently, the standard treatment consists of a combination of cisplatin and pemetrexed. The role of surgery and multimodality treatment remains controversial, while new treatment approaches, such as immunotherapy and targeted therapies, ad promising and interesting options. This review provides a comprehensive evaluation of emerging therapies and predictive biomarkers that are being tested.
\end{abstract}

Keywords Antineoplastic combined chemotherapy protocols/therapeutic use - Combined modality therapy · Drug resistance, neoplasm · Humans · Mesothelioma/drug therapy/pathology/surgery $\cdot$ Neoplasm staging ·

Pleural neoplasms/drug therapy/pathology/surgery .

Extrapleural pneumonectomy

\section{Introduction}

Malignant pleural mesothelioma (MPM) is an aggressive tumor posing major treatment challenges. Its widespread distribution on the pleural surface does not easily permit an adequate resection: a radical resection inevitably compromises a large amount of normal lung tissue. Furthermore,

\footnotetext{
J. M. M. F. Quispel-Janssen • P. Baas ( $₫)$

Department of Thoracic Oncology, The Netherlands Cancer

Institute- Antoni van Leeuwenhoek Hospital,

Plesmanlaan 121,

1066 CX Amsterdam, The Netherlands

e-mail: p.baas@nki.nl

J. M. M. F. Quispel-Janssen

e-mail:.jm.janssen@nki.nl
}

MPM is resistant to the vast majority of systemic anticancer drugs.

The development of novel therapeutic strategies is hampered by several factors. Assessment of disease extent is complicated as is illustrated by the various staging systems for MPM [1]. Due to this variability in staging, patient cohorts in trials are not entirely comparable, leading to heterogeneous study outcomes. To address this problem, the International Association for the Study of Lung Cancer (IASLC) and the International Mesothelioma Interest Group (IMIG) initiated the Prospective Staging Project in Malignant Pleural Mesothelioma. Recommendations are expected by January 2014.

The modification of RECIST improved response evaluation, but still lacks sensitivity for adequate response assessment [2]. Especially for thin tumor rinds, measurements are unreliable (Fig. 1). Use of volumetric assessment is under investigation and seems promising for improving both staging and response evaluation [3-5].

Furthermore, MPM is a relatively rare and heterogeneous disease. The tumor comprises different histological subtypes: epithelioid, sarcomatoid, and mixed (or biphasic), each of which are prognostically different. Recent pathologic studies have identified new prognostic factors like the pleomorphic type, which is considered a subtype of epithelioid mesothelioma, but has a prognosis similar to that of sarcomatoid MPM [6]. Furthermore, stratification for nuclear grade, determined by nuclear atypia and mitotic count, enabled discrimination between three prognostic groups in a series of 323 MPM cases [7]. Predictive biomarkers on the contrary, have not been identified. To date, no biomarker has proven to be sufficiently robust to apply in routine clinical practice. All of the above complicate validation of new therapeutic strategies in adequately powered randomized clinical trials. 

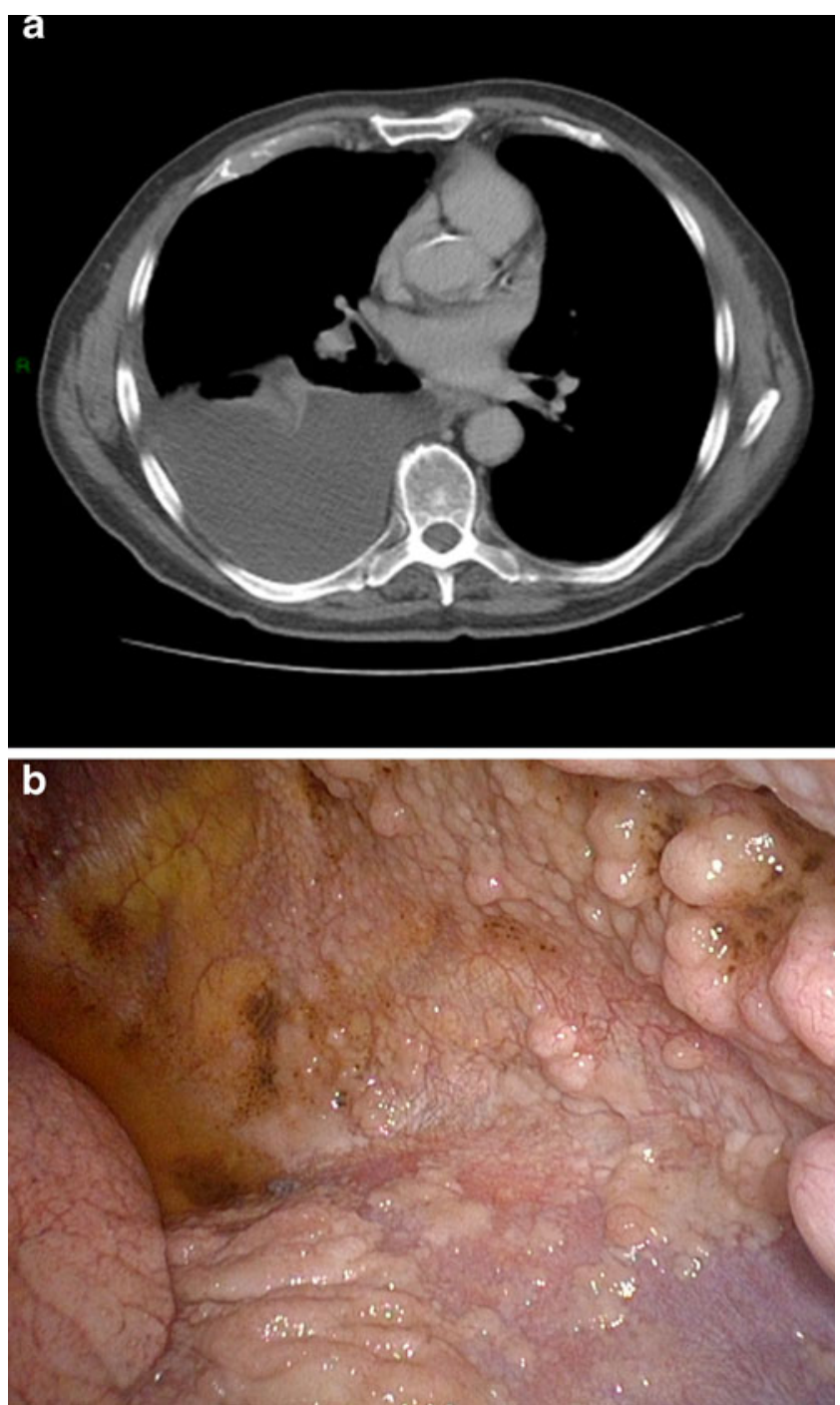

Fig. 1 a CT-scan showing a right-sided effusion in the thoracic cavity due to MPM. There is no clear measurable lesion. b Thoracoscopic image of the parietal pleura affected by MPM showing multiple small (2-4 mm) nodules

In this review, we provide a comprehensive overview of current treatment options and the research that is ongoing in MPM with a focus on predictive biomarkers.

\section{Surgery}

The role of surgery in MPM has been the subject of debate for many years. Cao et al. [8] systematically reviewed all literature on extrapleural pneumonectomy (EPP) up to 2010 and concluded that EPP as part of multimodality treatment, may improve survival in a group of highly selected patients. However, only few trials have addressed this issue prospectively $[9,10]$ and retrospective trials typically suffer from selection bias. Two recent major publications assessed feasibility of multimodality treatment in early-stage MPM. The MARS trial had patients undergo platinum-based induction chemotherapy and, if no signs of progression had occurred, randomized them to EPP followed by radiotherapy of the hemithorax, or to no EPP $[11,12 \bullet \cdot]$. The primary endpoint, feasibility of randomizing 50 patients within 1 year, was not met. Patients were accrued in a 3 -year time period. Only $45 \%$ of patients were eligible for randomization and only $33 \%$ of the randomized patients were able to complete the full trimodality treatment. Median overall survival (OS) in the EPP group was 18 months (calculated from start of chemotherapy) versus 23.1 months in the no EPP group. Toxicity was higher in the EPP group and quality of life was lower. In the EORTC phase II multicenter trial on trimodality therapy, "success of treatment" was the primary endpoint. This was defined as undergoing the full protocol treatment within defined time-frames and still being alive 90 days after end of treatment, progression-free, and without grade 3 or 4 toxicity [13*0]. Only $42.1 \%$ of patients fulfilled these criteria. Median OS of the whole group was 18.4 months, but in those who completed trimodality therapy, it was as high as 33 months. Ninety-day mortality was $6.5 \%$. Despite an encouraging 33 months' median survival, neither study favors EPP in MPM patients.

Pleurectomy/decortication (P/D) on the contrary, may play a role in MPM treatment. Flores et al. reported an improved survival in patients who underwent $\mathrm{P} / \mathrm{D}$, compared to those treated with EPP [14]. However, this study was retrospective and selection bias is likely. In addition, the definition and surgical techniques of pleurectomy and decortication, vary amongst different centers [15]. Prospective trials with a uniform definition of lung-sparing surgery for MPM are required to establish its role.

\section{Chemotherapy}

Since 2003, chemotherapy consisting of cisplatin and the antifolate pemetrexed is considered standard of care in MPM patients with an adequate performance status. Vogelzang et al. reported in their landmark study a response rate of $41 \%$ in patients treated with this combination [16]. Compared to cisplatin monotherapy, the combination arm demonstrated a survival benefit of approximately 3 months, leading to a 12 months median survival time. To reduce the hematologic toxicity of pemetrexed, supplementation of vitamin B12 and folic acid has proven its value [17]. Van Meerbeeck et al. reported similar progression-free survival (PFS) and OS results with raltitrexed, another anti-folate tested in a large randomized phase III EORTC trial combined with cisplatin [18]. Response rate, however, did not equal the cisplatin-pemetrexed combination $(24 \%$ vs $41 \%)$. Registration of raltitrexed for this indication has therefore been limited to a few European countries.

Carboplatin may be a reasonable substitute for cisplatin in MPM treatment. Ceresoli et al. reported a time to 
progression (TTP) of 6.5 months and median OS of 12.7 months in chemotherapy-naïve patients treated with carboplatin and pemetrexed [19].

Thymidylate synthase (TS), an enzyme involved in folate metabolism, was identified as a predictive biomarker for pemetrexed therapy. Righi et al. [20•] noted that low protein expression of TS predicted for better outcome in pemetrexedtreated MPM patients (TTP 17.9 vs 7.9 months and OS 30 vs 16.7 months). In order to confirm these retrospective data, a prospective randomized trial should be conducted. However, this is not feasible since approximately 1700 patients would be required per study arm to power such a trial. High expression of the excision repair cross-complementation group 1 (ERCC1) protein in this group of patients, was a prognostic but not a predictive marker [20 $0^{\bullet}$.

Anti-tumor activity of the gemcitabine-cisplatin combination was assessed in several phase II trials showing response rates between $12 \%$ and $48 \%$ [21-24]. Although never tested in a randomized phase III trial, this regimen demonstrated survival outcomes similar to the pemetrexedcisplatin combination in a retrospective study by Lee and coworkers [25].

\section{Second and further lines of treatment}

Studies in second-line treatment have yielded response rates between $10 \%$ and $20 \%$ with doxorubicin [26], pemetrexed alone $[27,28]$, pemetrexed in combination with carboplatin [28], vinorelbine [29], or cisplatin in combination with irinotecan and mitomycin [30]. A retrospective analysis of post-study treatment (PST) of patients included in the landmark study by Vogelzang indicated that PST was associated with a better survival, regardless of the choice of chemotherapy [31]. This may suggest a benefit of second or further lines of treatment in a subset of patients, although a clear survival benefit was not seen in any randomized trial [32••]. Retreatment with a pemetrexed-based regimen seems to be a valid option. A response rate of $19 \%$ has been noted in an observational study concerning patients that displayed an objective response or stable disease lasting for at least 3 months after first-line pemetrexed-based chemotherapy [33]. A similar response rate was observed in a second-line phase II trial of patients receiving biweekly gemcitabine and docetaxel [34]. With addition of granulocyte colony-stimulating factor (GCSF) to limit hematologic toxicity, this regimen proved to be well tolerated. Clinical activity of single-agent taxanes however, is lacking [35]. Surprisingly, gemcitabine combined with cisplatin did not elicit any objective responses in secondline setting in another phase II study. Disease control rate was $67 \%$, but toxicity was substantial with $35 \%$ of patients having grade 3 neutropenia and $47 \%$ having grade 3 or 4 thrombocytopenia [36].

\section{Maintenance therapy}

Only few studies have addressed the subject of maintenance therapy in MPM. A small single-arm phase II study by Van den Bogaert et al. reported pemetrexed maintenance therapy to be feasible and capable of evoking an ongoing response after induction chemotherapy [37]. The Cancer and Leukemia Group B (CALGB) currently runs a randomized phase II study, comparing maintenance pemetrexed to placebo in non-progressive patients after firstline chemotherapy, consisting of pemetrexed and cisplatin/ carboplatin. Progression-free survival was defined as the primary endpoint (data collection to be completed by January 2012) [38]. The histone deacetylase (HDAC) inhibitor vorinostat was investigated in maintenance setting and is discussed further on in this manuscript.

\section{Targeted therapies}

In recent years, research has focused on exploring the molecular pathways involved in growth and progression of MPM. Several drugs that target these pathways are being tested to define their role in MPM treatment (Table 1).

\section{Histone deacetylase inhibitors}

Epigenetic modifications such as hypermethylation and histone regulation play an important role in tumorigenesis. Histones are packaging proteins, clustering DNA to form chromatin. Gene transcription can only occur after decondensation of chromatin. Histone deacetylase (HDAC) inhibitors are a class of antitumor agents that modulate chromatin structure, thereby regulating gene transcription leading to apoptosis, inhibition of angiogenesis, and cell cycle arrest. Preclinical data have suggested a promising role for these agents in MPM [39, 40]. However, in a phase II study with HDAC inhibitor belinostat, no anti-tumour activity was noted [41]. Recently, the results of a large randomized phase III trial comparing HDAC inhibitor vorinostat to placebo in pretreated patients was presented at the ESMO conference in Stockholm. Despite encouraging response rates in an earlier phase I study [42], the randomized trial demonstrated only a minor improvement in PFS and no survival benefit at all (HR 0.98) [43]. Valproic acid, another HDAC inhibitor, was tested in combination with doxorubicin in recurrent MPM [44]. The response rate of $16 \%$ was higher than that of doxorubicin monotherapy [26]. These data do not support the use of the currently tested HDAC inhibitors in routine clinical practice. The role of HDAC inhibitors in combination with chemotherapy needs further evaluation. 


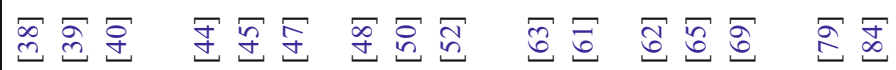<smiles>CCCC1CCCCC1</smiles>

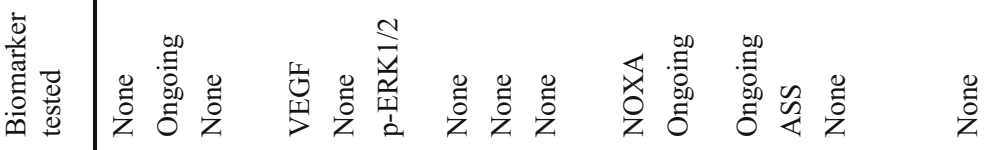

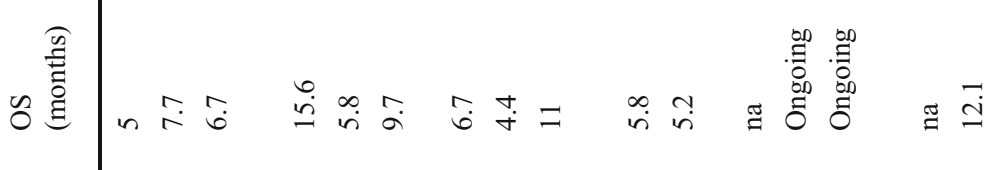

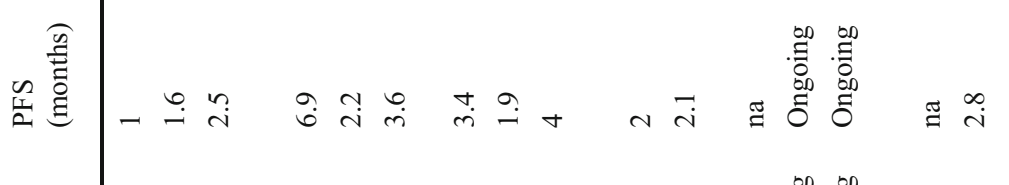

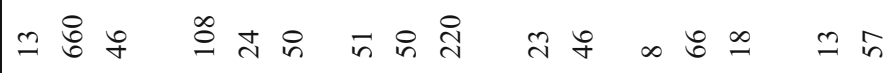

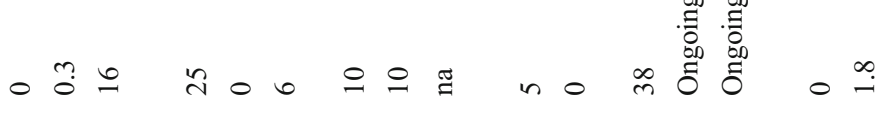

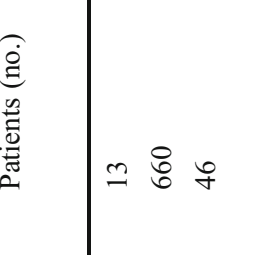

$\stackrel{2}{\circ}$

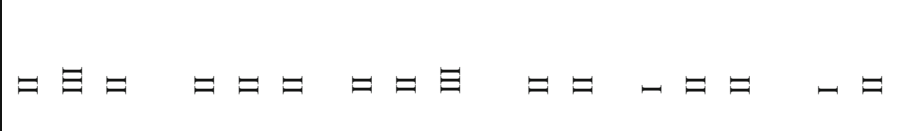

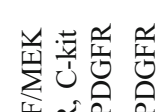

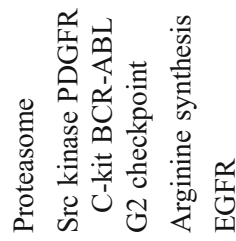

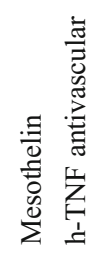


Anti-angiogenic agents

Angiogenesis, the process of new blood vessel formation, is essential for growth of solid tumors. Increase in angiogenesis, reflected by an increase in microvessel density (MVD), is a negative prognostic factor in MPM patients [45]. Several regulators of angiogenesis, such as vascular endothelial growth factor (VEGF), platelet-derived growth factor (PDGF), fibroblast growth factor-2 (FGF-2), and transforming growth factor beta (TGF- $\beta$ ), may serve as targets for treatment. VEGF is the most potent regulator of growth, and expression in MPM tissue is high compared to that in benign mesothelial cells [46].

Bevacizumab, a monoclonal antibody that neutralizes VEGF, is being investigated in combination with several chemotherapeutic regimens. Previous phase II trials did not report clinical activity of bevacizumab when added to standard chemotherapy [47] or EGFR-TKI [48]. Zalcman et al. described an increase in disease control rate in patients treated with bevacizumab and cisplatin and pemetrexed (73.5\% vs $43.2 \%$ in placebo) in a phase II study in previously untreated patients [49]. The final results of this and other trials have to be awaited to determine if bevacizumab has a role in the treatment of MPM.

Another method to block the VEGF pathway is to inhibit the tyrosine kinase activity of the VEGF receptor. Sorafenib targets the tyrosine kinase domain of both the VEGFreceptor and PDGF-receptor and inhibits the RAS/RAF/ MEK/ERK pathway. A phase II study of sorafenib as single agent in 50 chemotherapy-naïve or pretreated MPM patients showed a limited response rate of $6 \%$. Median PFS and OS were 3.6 and 9.7 months, respectively. Low or negative phosphorylation status of ERK1/2 in tumor tissue was correlated with improved survival [50].

Sunitinib, another VEGF-receptor and PDGF-receptor tyrosine kinase inhibitor (TKI), was tested in 53 previously treated MPM patients. Response rate was assessed by modified RECIST criteria on CT-scan and by metabolic response on FDG-PET. The total response rate was $22 \%$, with $10 \%$ of the responses confirmed by modified RECIST on CT [51]. Metabolic response on FDG-PET may be a more accurate way than modified RECIST to assess response, but its clinical relevance remains to be proven. In this study, however, the median TTP (3.4 months) and median OS (6.7 months) do not support the claim of modest activity. Furthermore, toxicity required dose reductions in $28 \%$ of patients. Another phase II study confirms the lack of clinical activity of sunitinib as single agent [52].

Campbell and coworkers presented their results of a phase II study involving cediranib at the latest ASCO annual meeting. This tyrosine kinase inhibitor of VEGFR and PDGFR was poorly tolerated requiring dose reductions in $48 \%$ and discontinuation for toxicity in $26 \%$ of patients. The trial failed to meet its prespecified response endpoint with a response rate of $10 \%$ [53].

Thalidomide is an immunomodulating drug that also acts on promoter regions of growth factor genes such as VEGF and FGF-2 by intercalating into guanine $(\mathrm{G})$ and cytosine (C) rich regions of DNA. Subsequently, VEGF and FGF expression levels decrease, thereby diminishing angiogenesis and tumor growth. After promising results from a phase I study in 40 MPM patients [54], a multicenter, randomized phase III study comparing thalidomide maintenance therapy to observation was launched. In this large trial, 222 patients without disease progression after induction chemotherapy were included. Despite only mild toxicity, there was no benefit of thalidomide in PFS or OS [55].

So far, clinical activity of anti-angiogenic drugs is disappointing. Two major mechanisms of resistance to these drugs have been suggested by Bergers and Hanahan. Firstly, intrinsic resistance is determined by specific tumor microenvironment, and secondly, evasive resistance is due to upregulation of alternative pro-angiogenic pathways [56]. A strategy to combine anti-angiogenic drugs with targeted agents might be a way to move forward. For this we need predictive biomarkers for response or resistance. Furthermore, it is essential to get a better understanding of the processes that evolve during treatment. Therefore, we developed a study protocol with interim biopsy analysis for a randomized phase II trial combining cisplatin and pemetrexed with axitinib, a VEGFR and PDGFR TKI, or placebo [57]. So far, patient accrual is satisfactory and performing a second thoracoscopy for interim biopsy analysis is feasible. Results of this study are awaited in 2012.

\section{$\mathrm{PI} 3 \mathrm{~K} / \mathrm{AKT} / \mathrm{mTOR}$ pathway}

The PI3K/AKT/mTOR pathway is involved in a number of cellular processes that regulate proliferation, survival, and motility [58]. In MPM this pathway is frequently dysregulated which makes it an interesting target for therapy [59]. Several PI3K inhibitors are currently being developed and a randomized phase III study in recurrent MPM patients is in preparation. The downstream effector of this pathway, mTOR, can be inhibited by agents like sirolimus, temsirolimus, and everolimus, currently used as immune suppressors in transplantation medicine. Everolimus is being tested in a phase II trial in MPM patients with disease recurrence. Loss of Merlin/NF2 will be evaluated as a biomarker to predict anti-tumour activity [60]. The Southwest Oncology Group (SWOG) is also evaluating everolimus in recurrent MPM [61].

Other targeted agents

Bortezomib is a selective proteasome inhibitor that decreases nuclear factor- $\mathrm{\kappa} \mathrm{B}$ and upregulates proapoptotic $\mathrm{BH} 3$ proteins. 
A single-agent phase II trial has evaluated efficacy of this drug in first and second line setting. As clinical activity is lacking, further investigation as monotherapy is not warranted (Fennell et al., submitted). The association of NOXA expression to response was assessed in this trial, showing that NOXA cannot be used as a predictive biomarker. Two trials regarding bortezomib are ongoing: one combining it to cisplatin [62] and the other to oxaliplatin [63].

Dasatinib, a receptor TKI of Src family kinases, PDGFR, C-kit, and BCR-ABL fusion protein, did not show activity in MPM and was poorly tolerated [64]. Data on pretreatment and post-treatment plasma levels of several biomarkers will be available in due time.

Tumor cells that acquire DNA damage usually arrest cell cycles to repair damaged DNA. Most solid tumors have genetic alterations that disturb cell cycle checkpoint G1 which makes them dependent on checkpoint G2 for survival. CBP501 is a compound that abrogates the G2 checkpoint, resulting in tumor cell death. This compound has demonstrated promising activity in combination with cisplatin in patients with MPM and patients with ovarian cancer in a phase I trial. Three out of 8 MPM patients showed a response. In two of them, time to progression was more than 9 months. Doselimiting toxicity (DLT) consisted of a histamine-release syndrome [65]. A phase II study with CBP501 in combination with cisplatin and pemetrexed is currently recruiting patients with MPM.

Arginine is an amino acid involved in tumor metabolism and essential for tumor growth. Arginine synthesis is regulated by the enzyme argininosuccinate synthetase (ASS) and is downregulated in a number of tumor types such as MPM, hepatocellular carcinoma, and melanoma. Loss of ASS results in dependence on extracellular arginine. In a study by Szlosarek et al., 63\% of mesothelioma patients had reduced or absent levels of ASS [66]. Pegylated arginine deiminase (ADI-PEG 20) is an arginine-depleting drug that has demonstrated interesting results in a phase I/II study in hepatocellular carcinoma and melanoma [67]. A multicenter randomized phase II of single agent ADI-PEG 20TM was recently launched in MPM patients with ASS-negative tumors [68]. ASS expression may serve as a biomarker predictive for treatment response of ADI-PEG 20.

The epidermal growth factor receptor (EGFR) is overexpressed in more than $50 \%$ of MPM patients. Activating mutations in the EGF receptor, however, are not prevalent in MPM [69]. This is reflected by the lack of activity of EGFR-tyrosine kinase inhibitors gefitinib and erlotinib in patients with MPM [70, 71]. Cetuximab is a monoclonal antibody binding to the EGF-receptor that has shown a survival benefit in non-small cell lung cancer (NSCLC) patients with high EGFR expression [72]. A study exploring the role of cetuximab in combination with pemetrexed and cisplatin or carboplatin is ongoing [73].

\section{Immunotherapy}

Immunotherapy may be an attractive treatment approach for MPM for several reasons. The large lymphocyte infiltrate present in many cases of mesothelioma, and the spontaneous regression, occasionally occurring in MPM, suggest a role for the immune system in controlling tumor growth. Furthermore, several tumor-stroma generated cytokines (eg, TGF- $\beta$ ) suppress the local immune system, as do the abundant regulatory $\mathrm{T}$ cells in MPM [74]. In the past, various passive immunotherapeutic approaches with cytokines such as IL-2, IL-12, INF- $\beta$, and INF- $\gamma$ were tested in murine models $[75,76]$ and some even in phase I-II clinical trials but with limited success [77-79]. Hegmans et al. previously demonstrated efficacy of active immunotherapy in a murine MPM model using tumor lysate-pulsed dendritic cell vaccination [80]. Recently, the results of a phase I trial testing this dendritic cell-based (DC) immunotherapy were published. Ten patients received three vaccinations after completing standard chemotherapy. DC immunotherapy is feasible, well-tolerated, and capable of inducing an immunological response to mesothelioma cells $[81 \bullet \bullet]$. It seems most effective in patients with modest tumor load. Applying DC immunotherapy after surgical debulking is an interesting approach for future studies. A trial combining DC immunotherapy with cyclophosphamide, inhibiting Tregulatory lymphocytes and thereby enhancing immunological responses, is currently recruiting patients [82].

Mesothelin is a glycoprotein normally expressed on the surface of mesothelial cells lining the pleural and peritoneal cavity. Expression is upregulated in many solid tumors including MPM. Mesothelin can bind to CA-125, a cell surface mucin expressed on several types of tumor cells, thereby mediating tumor metastasis within pleural and peritoneal cavities [83]. At least two different antibodies that target mesothelin were developed and tested in phase I trials. MORAb-009 is a chimeric monoclonal antibody to mesothelin that was well tolerated and induced disease stabilization in patients with mesothelin-expressing tumors [84]. An open-label clinical trial of MORAb-009 in combination with pemetrexedcisplatin in patients with MPM has completed accrual and results are awaited [85]. SS1P (CAT-5001) is a recombinant immunotoxin linking an exotoxin of Pseudomonas aeruginosa to mesothelin. Tolerability was demonstrated previously in a phase I study [86]. Currently it is being tested in combination with cisplatin and pemetrexed in MPM patients [87]. Another phase I study is combining SS1P with an immunedepleting regimen consisting of pentostatin and cyclophosphamide [88].

Tumor necrosis factor $\alpha(\mathrm{TNF}-\alpha)$ is a potent anti-tumour agent. Systemic use, however, is limited by severe toxicity [89]. Asparagine-glycine-arginine-human tumor necrosis factor- $\alpha$ (NGR-hTNF) is a fusion protein of human TNF- $\alpha$ and asparagine-glycine-arginine, a peptide that targets 
aminopeptidase N/CD13. This aminopeptidase N/CD13 is overexpressed by endothelial cells of the majority of solid tumors [90]. NGR-hTNF was tested as single agent in triweekly and weekly dosing in MPM patients with disease recurrence. NGR-hTNF was well tolerated, with short-lived chills being the most common side effects. Progression-free survival was 2.8 months and OS 12.1 months [91]. A randomized double-blind phase II maintenance study of NGR-hTNF versus placebo is currently recruiting patients with advanced MPM [92]. A phase III study is also initiated comparing NGR-hTNF plus chemotherapy (best investigators' choice $[\mathrm{BIC}]$ ) to placebo in combination with chemotherapy BIC in patients previously treated with pemetrexed [93].

\section{Gene therapy}

The purpose of gene therapy is to kill tumor cells by means of genetic modification. In general this implies that a therapeutic gene is inserted into tumor cells using a vector system. Several viruses such as adenovirus or vacciniavirus may serve as such. In MPM the vector can be administered locally via the pleural cavity. The inserted gene can either be a suicide or sensitivity gene (eg, herpes simplex virus thymidine kinase), an immune modulator (eg, IL-6 or IFN- $\beta$ ), or a replacement for a tumor suppressor gene. Sterman et al. recently published their results of intrapleural administration of an adenoviral vector expressing interferon $\beta$ [94]. Ten patients were treated with an intrapleural injection which was repeated after 1 week. Gene transfer was confirmed in the pleural fluid. One patient had a partial response and two patients disease stabilization. However, neutralizing antibodies were rapidly developed after the first dose, preventing effective gene transfer. An early second injection after 3 days is currently being tested.

\section{Conclusions and future perspectives}

Despite ceaseless efforts to improve outcome in patients with MPM, the prognosis remains grim. The standard of care consisting of cisplatin-pemetrexed chemotherapy has not changed since 2003. Surgery should not be advocated outside clinical trials and targeted therapies have not entered clinical practice yet, due to lack of activity. In order to improve prognosis, several measures are necessary. Firstly, we have to reconsider our current classification based on epithelioid versus non-epithelioid histology. Secondly, an improved system for staging and response assessment is required. In addition, we need better criteria to select patients that may benefit from surgery. The same applies to patient selection for targeted therapies, as biomarkers predicting for treatment response are urgently needed. Furthermore, preclinical data suggest that in approximately half of MPM cases, more than one pathway is activated [95]. Therefore, combining targeted agents is a treatment strategy worth exploring. Finally, to get a better understanding of the pathways involved in tumorigenesis, we advocate combining clinical trials with translational research.

Disclosure No potential conflicts of interest relevant to this article were reported.

\section{References}

Papers of particular interest, published recently, have been highlighted as:

- Of importance

•- Of major importance

1. Van Schil P. Malignant pleural mesothelioma: staging systems. Lung Cancer. 2005;49 Suppl 1:S45-8.

2. Byrne MJ, Nowak AK. Modified RECIST criteria for assessment of response in malignant pleural mesothelioma. Ann Oncol. 2004;15(2):257-60.

3. Ak G, Metintas M, Metintas S, et al. Three-dimensional evaluation of chemotherapy response in malignant pleural mesothelioma. Eur J Radiol. 2010;74(1):130-5.

4. Liu F, Zhao B, Krug LM, et al. Assessment of therapy responses and prediction of survival in malignant pleural mesothelioma through computer-aided volumetric measurement on computed tomography scans. J Thorac Oncol. 2010;5(6):879-84.

5. Frauenfelder T, Tutic M, Weder W, et al. Volumetry: an alternative to assess therapy response for malignant pleural mesothelioma? Eur Respir J. 2011;38(1):162-8.

6. Kadota K, Suzuki K, Sima CS, et al. Pleomorphic epithelioid diffuse malignant pleural mesothelioma: a clinicopathological review and conceptual proposal to reclassify as biphasic or sarcomatoid mesothelioma. J Thorac Oncol. 2011;6(5):896-904.

7. Kadota K, Suzuki K, Colovos C, et al. A nuclear grading system is a strong predictor of survival in epitheloid diffuse malignant pleural mesothelioma. Mod Pathol. 2012;25(2):260-71.

8. Cao CQ, Yan TD, Bannon PG, et al. A systematic review of extrapleural pneumonectomy for malignant pleural mesothelioma. J Thorac Oncol. 2010;5(10):1692-703.

9. Weder W, Stahel RA, Bernhard J, et al. Multicenter trial of neoadjuvant chemotherapy followed by extrapleural pneumonectomy in malignant pleural mesothelioma. Ann Oncol. 2007;18(7):1196-202.

10. Krug LM, Pass HI, Rusch VW, et al. Multicenter phase II trial of neoadjuvant pemetrexed plus cisplatin followed by extrapleural pneumonectomy and radiation for malignant pleural mesothelioma. J Clin Oncol. 2009;27(18):3007-13.

11. Treasure T, Waller D, Tan C, et al. The Mesothelioma and Radical surgery randomized controlled trial: the Mars feasibility study. J Thorac Oncol. 2009;4(10):1254-8.

12. •• Treasure T, Lang-Lazdunski L, Waller D, et al. Extra-pleural pneumonectomy versus no extra-pleural pneumonectomy for patients with malignant pleural mesothelioma: clinical outcomes of the Mesothelioma and Radical Surgery (MARS) randomised feasibility study. Lancet Oncol. 2011;12(8):763-72. This large study defines the role of extrapleural pneumonectomy as part of trimodality treatment in MPM.. 
13. • Van Schil PE, Baas P, Gaafar R, et al. Trimodality therapy for malignant pleural mesothelioma: results from an EORTC phase II multicentre trial. Eur Respir J. 2010;36(6):1362-9. This important publication also defines the role of extrapleural pneumonectomy as part of trimodality treatment in $M P M$..

14. Flores RM, Pass HI, Seshan VE, et al. Extrapleural pneumonectomy versus pleurectomy/decortication in the surgical management of malignant pleural mesothelioma: results in 663 patients. J Thorac Cardiovasc Surg. 2008;135(3):620-6. 6 e1-3.

15. Teh E, Fiorentino F, Tan C, et al. A systematic review of lungsparing extirpative surgery for pleural mesothelioma. J R Soc Med. 2011;104(2):69-80.

16. Vogelzang NJ, Rusthoven JJ, Symanowski J, et al. Phase III study of pemetrexed in combination with cisplatin versus cisplatin alone in patients with malignant pleural mesothelioma. J Clin Oncol. 2003;21(14):2636-44.

17. Scagliotti GV, Shin DM, Kindler HL, et al. Phase II study of pemetrexed with and without folic acid and vitamin B12 as front-line therapy in malignant pleural mesothelioma. J Clin Oncol. 2003;21(8):1556-61.

18. van Meerbeeck JP, Gaafar R, Manegold C, et al. Randomized phase III study of cisplatin with or without raltitrexed in patients with malignant pleural mesothelioma: an intergroup study of the European Organisation for Research and Treatment of Cancer Lung Cancer Group and the National Cancer Institute of Canada. J Clin Oncol. 2005;23(28):6881-9.

19. Ceresoli GL, Zucali PA, Favaretto AG, et al. Phase II study of pemetrexed plus carboplatin in malignant pleural mesothelioma. J Clin Oncol. 2006;24(9):1443-8.

20. • Righi L, Papotti MG, Ceppi P, et al. Thymidylate synthase but not excision repair cross-complementation group 1 tumor expression predicts outcome in patients with malignant pleural mesothelioma treated with pemetrexed-based chemotherapy. J Clin Oncol. 2010;28(9):1534-9. This study describes a predictive biomarker for pemetrexed treatment.

21. Byrne MJ, Davidson JA, Musk AW, et al. Cisplatin and gemcitabine treatment for malignant mesothelioma: a phase II study. J Clin Oncol. 1999;17(1):25-30.

22. Nowak AK, Byrne MJ, Williamson R, et al. A multicentre phase II study of cisplatin and gemcitabine for malignant mesothelioma. $\mathrm{Br}$ J Cancer. 2002;87(5):491-6.

23. van Haarst JM, Baas P, Manegold C, et al. Multicentre phase II study of gemcitabine and cisplatin in malignant pleural mesothelioma. Br J Cancer. 2002;86(3):342-5.

24. Kalmadi SR, Rankin C, Kraut MJ, et al. Gemcitabine and cisplatin in unresectable malignant mesothelioma of the pleura: a phase II study of the Southwest Oncology Group (SWOG 9810). Lung Cancer. 2008;60(2):259-63.

25. Lee CW, Murray N, Anderson H, et al. Outcomes with first-line platinum-based combination chemotherapy for malignant pleural mesothelioma: a review of practice in British Columbia. Lung Cancer. 2009;64(3):308-13.

26. Harvey VJ, Slevin ML, Ponder BA, et al. Chemotherapy of diffuse malignant mesothelioma. Phase II trials of single-agent 5-fluorouracil and adriamycin. Cancer. 1984;54(6):961-4.

27. Jassem J, Ramlau R, Santoro A, et al. Phase III trial of pemetrexed plus best supportive care compared with best supportive care in previously treated patients with advanced malignant pleural mesothelioma. J Clin Oncol. 2008;26(10):1698-704.

28. Sorensen JB, Sundstrom S, Perell K, et al. Pemetrexed as secondline treatment in malignant pleural mesothelioma after platinumbased first-line treatment. J Thorac Oncol. 2007;2(2):147-52.

29. Stebbing J, Powles T, McPherson K, et al. The efficacy and safety of weekly vinorelbine in relapsed malignant pleural mesothelioma. Lung Cancer. 2009;63(1):94-7.
30. Fennell DA, Steele JP, Shamash J, et al. Efficacy and safety of first- or second-line irinotecan, cisplatin, and mitomycin in mesothelioma. Cancer. 2007;109(1):93-9.

31. Manegold C, Symanowski J, Gatzemeier U, et al. Second-line (post-study) chemotherapy received by patients treated in the phase III trial of pemetrexed plus cisplatin versus cisplatin alone in malignant pleural mesothelioma. Ann Oncol. 2005;16(6):923-7.

32. •- Scherpereel A, Astoul P, Baas P, et al. Guidelines of the European Respiratory Society and the European Society of Thoracic Surgeons for the management of malignant pleural mesothelioma. Eur Respir J. 2010;35(3):479-95. This paper presents the European guidelines for diagnosis and treatment of MPM.

33. Ceresoli GL, Zucali PA, De Vincenzo F, et al. Retreatment with pemetrexed-based chemotherapy in patients with malignant pleural mesothelioma. Lung Cancer. 2011;72(1):73-7.

34. Tourkantonis I, Makrilia N, Ralli M, et al. Phase II study of gemcitabine plus docetaxel as second-line treatment in malignant pleural mesothelioma: a single institution study. Am J Clin Oncol. 2011;34(1):38-42.

35. Belani CP, Adak S, Aisner S, et al. Docetaxel for malignant mesothelioma: phase II study of the Eastern Cooperative Oncology Group. Clin Lung Cancer. 2004;6(1):43-7.

36. Pasello G, Nicotra S, Marulli G, et al. Platinum-based doublet chemotherapy in pre-treated malignant pleural mesothelioma (MPM) patients: a mono-institutional experience. Lung Cancer. 2011;73(3):351-5.

37. van den Bogaert DP, Pouw EM, van Wijhe G, et al. Pemetrexed maintenance therapy in patients with malignant pleural mesothelioma. J Thorac Oncol. 2006;1(1):25-30.

38. Pemetrexed Disodium or Observation in Treating Patients With Malignant Pleural Mesothelioma Without Progressive Disease After First-Line Chemotherapy. ClinicalTrials.gov Identifier: NCT01085630 Available from: http://clinicaltrials.gov.

39. Vandermeers F, Hubert P, Delvenne P, et al. Valproate, in combination with pemetrexed and cisplatin, provides additional efficacy to the treatment of malignant mesothelioma. Clin Cancer Res. 2009;15(8):2818-28.

40. Symanowski J, Vogelzang N, Zawel L, et al. A histone deacetylase inhibitor LBH589 downregulates XIAP in mesothelioma cell lines which is likely responsible for increased apoptosis with TRAIL. J Thorac Oncol. 2009;4(2):149-60.

41. Ramalingam SS, Belani CP, Ruel C, et al. Phase II study of belinostat (PXD101), a histone deacetylase inhibitor, for second line therapy of advanced malignant pleural mesothelioma. J Thorac Oncol. 2009;4(1):97-101.

42. Kelly WK, O'Connor OA, Krug LM, et al. Phase I study of an oral histone deacetylase inhibitor, suberoylanilide hydroxamic acid, in patients with advanced cancer. J Clin Oncol. 2005;23(17):3923-31.

43. Krug LM, Kindler H, Calvert H, et al. VANTAGE 014: Vorinostat in patients with advanced malignant pleural mesothelioma who have failed prior pemetrexed and either cisplatin or carboplatin therapy: a phase III randomized double-blind, placebo-controlled trial. 2011 European Multidisciplinary Cancer Congress [abstract 3BA]: Eur J Cancer. Vol 47, Suppl 2.

44. Scherpereel A, Berghmans T, Lafitte JJ, et al. Valproatedoxorubicin: promising therapy for progressing mesothelioma. A phase II study. Eur Respir J. 2011;37(1):129-35.

45. Edwards JG, Cox G, Andi A, et al. Angiogenesis is an independent prognostic factor in malignant mesothelioma. Br J Cancer. 2001;85 (6):863-8.

46. Kumar-Singh S, Weyler J, Martin MJ, et al. Angiogenic cytokines in mesothelioma: a study of VEGF, FGF-1 and -2, and TGF beta expression. J Pathol. 1999;189(1):72-8.

47. Karrison T, Kindler HL, Gandara DR, et al. Final analysis of a multi-center, double-blind, placebo-controlled, randomized phase II trial of gemcitabine/cisplatin plus bevacizumab or placebo in 
patients with malignant mesothelioma. 2007 ASCO Annual Meeting: J of Clin Oncol, Vol 25, No. 18S (June 20 Supplement); [abstract 7526].

48. Jackman DM, Kindler HL, Yeap BY, et al. Erlotinib plus bevacizumab in previously treated patients with malignant pleural mesothelioma. Cancer. 2008;113(4):808-14.

49. Zalcman G, Margery J, Scherpereel A, et al, editors. IFCTGFPC-0701 MAPS trial, a multicenter randomized phase II/III trial of pemetrexed-cisplatin with or without bevacizumab in patients with malignant pleural mesothelioma. 2010 ASCO Annual Meeting; [abstract 7020]: J Clin Oncol. Vol 28, Suppl 15.

50. Dubey S, Janne PA, Krug L, et al. A phase II study of sorafenib in malignant mesothelioma: results of Cancer and Leukemia Group B 30307. J Thorac Oncol. 2010;5(10):1655-61.

51. Nowak AK, Millward MJ, Francis RJ, et al., editors. Final results of a phase II study of sunitinib as second-line therapy in malignant pleural mesothelioma (MPM). 2010 ASCO Annual Meeting; [abstract 7036]: J Clin Oncol. Vol 28, Suppl 15.

52. Laurie SA, Gupta A, Chu Q, et al. Brief report: a phase II study of sunitinib in malignant pleural mesothelioma. the NCIC Clinical Trials Group. J Thorac Oncol. 2011;6(11):1950-4.

53. Campbell NP, Kunnavakkam R, Leighl NB, et al. editors. Cediranib (C) in patients (pts) with malignant mesothelioma (MM): A phase II trial of The University of Chicago Phase II Consortium. 2011ASCO Annual Meeting; [abstract 7027]: J Clin Oncol. Vol 29, suppl 15.

54. Baas P, Boogerd W, Dalesio O, et al. Thalidomide in patients with malignant pleural mesothelioma. Lung Cancer. 2005;48(2):291-6.

55. Baas P, Buikhuisen W, Dalesio O, et al., editors. A multicenter, randomized phase III maintenance study of thalidomide ( $\operatorname{arm} A)$ versus observation ( $\operatorname{arm} B$ ) in patients with malignant pleural mesothelioma (MPM) after induction chemotherapy. 2011 ASCO Annual Meeting [abstract 7006]: J Clin Oncol. Vol 29, suppl 15.

56. Bergers G, Hanahan D. Modes of resistance to anti-angiogenic therapy. Nat Rev Cancer. 2008;8(8):592-603.

57. Axitinib in Malignant Mesothelioma (N08CPA). ClinicalTrials.gov Identifier: NCT01211275 Available from: http://www.clinicaltrials. gov.

58. Manning BD, Cantley LC. AKT/PKB signaling: navigating downstream. Cell. 2007;129(7):1261-74.

59. Suzuki Y, Murakami H, Kawaguchi K, et al. Activation of the PI3K-AKT pathway in human malignant mesothelioma cells. Mol Med Report. 2009;2(2):181-8.

60. Everolimus (RAD001) for the Treatment of Malignant Pleural Mesothelioma With Merlin/NF2 Loss as a Biomarker to Predict Sensitivity. ClinicalTrials.gov Identifier: NCT01024946 Available from: http://www.clinicaltrials.gov.

61. Everolimus in Treating Patients With Pleural Malignant Mesothelioma That Cannot Be Removed By Surgery. ClinicalTrials.gov Identifier: NCT00770120 Available from: http://www.clinicaltrials. gov.

62. Bortezomib and Cisplatin as First-Line Therapy in Treating Patients With Malignant Mesothelioma. ClinicalTrials.gov Identifier: NCT00458913 Available from: http://www.clinicaltrials. gov.

63. Velcade and Eloxatin for Patients With Malignant Pleural or Peritoneal Mesothelioma. ClinicalTrials.gov Identifier: NCT00996385 Available from: http://www.clinicaltrials.gov.

64. Dudek A, Pang H, Kratzke RA, et al., editors. CALGB 30601: A phase II study of dasatinib (D) in patients (pts) with previously treated malignant mesothelioma (MM). 2010 ASCO Annual Meeting J Clin Oncol. Vol 28, Suppl 15.

65. Shapiro GI, Tibes R, Gordon MS, et al. Phase I studies of CBP501, a G2 checkpoint abrogator, as monotherapy and in combination with cisplatin in patients with advanced solid tumors. Clin Cancer Res. 2011;17(10):3431-42.

66. Szlosarek PW, Klabatsa A, Pallaska A, et al. In vivo loss of expression of argininosuccinate synthetase in malignant pleural mesothelioma is a biomarker for susceptibility to arginine depletion. Clin Cancer Res. 2006;12(23):7126-31.

67. Delage B, Fennell DA, Nicholson L, et al. Arginine deprivation and argininosuccinate synthetase expression in the treatment of cancer. Int J Cancer. 2010;126(12):2762-72.

68. A Clinical Trial of ADI-PEG 20TM in Patients With Malignant Pleural Mesothelioma (ADAM). ClinicalTrials.gov Identifier: NCT01279967 Available from: http://www.clinicaltrials.gov.

69. Cortese JF, Gowda AL, Wali A, et al. Common EGFR mutations conferring sensitivity to gefitinib in lung adenocarcinoma are not prevalent in human malignant mesothelioma. Int $\mathrm{J}$ Cancer. 2006;118(2):521-2.

70. Govindan R, Kratzke RA, Herndon 2nd JE, et al. Gefitinib in patients with malignant mesothelioma: a phase II study by the Cancer and Leukemia Group B. Clin Cancer Res. 2005;11 (6):2300-4

71. Garland LL, Rankin C, Gandara DR, et al. Phase II study of erlotinib in patients with malignant pleural mesothelioma: a Southwest Oncology Group Study. J Clin Oncol. 2007;25(17):2406-13.

72. Pirker R, Pereira JR, von Pawel J, et al. EGFR expression as a predictor of survival for first-line chemotherapy plus cetuximab in patients with advanced non-small-cell lung cancer: analysis of data from the phase 3 FLEX study. Lancet Oncol. 2011 Nov 3.

73. A Study of Cetuximab Combined With Cisplatin or Carboplatin/ Pemetrexed as First Line Treatment in Patients With Malignant Pleural Mesothelioma. (MesoMab). ClinicalTrials.gov Identifier: NCT00996567 Available from: http://www.clinicaltrials.gov.

74. Gregoire M. What's the place of immunotherapy in malignant mesothelioma treatments? Cell Adh Migr. 2010;4(1):15361.

75. Jackaman C, Bundell CS, Kinnear BF, et al. IL-2 intratumoral immunotherapy enhances $\mathrm{CD} 8+\mathrm{T}$ cells that mediate destruction of tumor cells and tumor-associated vasculature: a novel mechanism for IL-2. J Immunol. 2003;171(10):5051-63.

76. Caminschi I, Venetsanakos E, Leong CC, et al. Cytokine gene therapy of mesothelioma. Immune and antitumor effects of transfected interleukin-12. Am J Respir Cell Mol Biol. 1999;21(3):34756.

77. Astoul P, Viallat JR, Laurent JC, et al. Intrapleural recombinant IL2 in passive immunotherapy for malignant pleural effusion. Chest. 1993;103(1):209-13.

78. Christmas TI, Manning LS, Garlepp MJ, et al. Effect of interferonalpha 2a on malignant mesothelioma. J Interferon Res. 1993;13 (1):9-12.

79. Boutin C, Nussbaum E, Monnet I, et al. Intrapleural treatment with recombinant gamma-interferon in early stage malignant pleural mesothelioma. Cancer. 1994;74(9):2460-7.

80. Hegmans JP, Hemmes A, Aerts JG, et al. Immunotherapy of murine malignant mesothelioma using tumor lysate-pulsed dendritic cells. Am J Respir Crit Care Med. 2005;171(10):1168-77.

81. • Hegmans JP, Veltman JD, Lambers ME, et al. Consolidative dendritic cell-based immunotherapy elicits cytotoxicity against malignant mesothelioma. Am J Respir Crit Care Med. 2010;181 (12):1383-90. This interesting article describes the promising results of dendritic cell-based immunotherapy.

82. Dendritic Cell-based Immunotherapy Combined With Low-dose Cyclophosphamide in Patients With Malignant Mesothelioma. ClinicalTrials.gov Identifier: NCT01241682 Available from: http://www.clinicaltrials.gov.

83. Rump A, Morikawa Y, Tanaka M, et al. Binding of ovarian cancer antigen CA125/MUC16 to mesothelin mediates cell adhesion. J Biol Chem. 2004;279(10):9190-8. 
84. Hassan R, Cohen SJ, Phillips M, et al. Phase I clinical trial of the chimeric anti-mesothelin monoclonal antibody MORAb-009 in patients with mesothelin-expressing cancers. Clin Cancer Res. 2010;16(24):6132-8.

85. An Efficacy Study of MORAb-009 Amatuximab in Subjects With Pleural Mesothelioma. ClinicalTrials.gov Identifier: NCT00738582 Available from: http://www.clinicaltrials.gov.

86. Hassan R, Bullock S, Premkumar A, et al. Phase I study of SS1P, a recombinant anti-mesothelin immunotoxin given as a bolus I.V. infusion to patients with mesothelin-expressing mesothelioma, ovarian, and pancreatic cancers. Clin Cancer Res. 2007;13 (17):5144-9.

87. SS1(dsFV)PE38 Plus Pemetrexed and Cisplatin to Treat Malignant Pleural Mesothelioma. ClinicalTrials.gov Identifier: NCT01445392; Available from: http://www.clinicaltrials.gov.

88. SS1P and Pentostatin Plus Cyclophosphamide for Mesothelioma. ClinicalTrials.gov Identifier: NCT01362790 Available from: http:// www.clinicaltrials.gov.

89. Gamm H, Lindemann A, Mertelsmann R, et al. Phase I trial of recombinant human tumour necrosis factor alpha in patients with advanced malignancy. Eur J Cancer. 1991;27(7):856-63.

90. Curnis F, Sacchi A, Borgna L, et al. Enhancement of tumor necrosis factor alpha antitumor immunotherapeutic properties by targeted delivery to aminopeptidase N (CD13). Nat Biotechnol. 2000;18(11):1185-90.

91. Gregorc V, Zucali PA, Santoro A, et al. Phase II study of asparagine-glycine-arginine-human tumor necrosis factor alpha, a selective vascular targeting agent, in previously treated patients with malignant pleural mesothelioma. J Clin Oncol. 2010;28 (15):2604-11.

92. Phase II Study of NGR-hTNF Versus Placebo as Maintenance Treatment in Patients With Advanced Malignant Pleural Mesothelioma (NGR019). ClinicalTrials.gov Identifier: NCT01358084 Available from: http://www.clinicaltrials.gov.

93. NGR015: Study in Second Line for Patient With Advanced Malignant Pleural Mesothelioma Pretreated With Pemetrexed. ClinicalTrials.gov Identifier: NCT01098266 Available from: http://www. clinicaltrials.gov.

94. Sterman DH, Haas A, Moon E, et al. A Trial of Intrapleural Adenoviral-mediated Interferon-\{alpha\}2b Gene Transfer for Malignant Pleural Mesothelioma. Am J Respir Crit Care Med. 2011 Jun 3.

95. Brevet M, Shimizu S, Bott MJ, et al. Coactivation of receptor tyrosine kinases in malignant mesothelioma as a rationale for combination targeted therapy. J Thorac Oncol. 2011;6(5):86474. 